\title{
Overview of State Estimation Technique for Power System Control
}

\author{
Ankhi Gulati ${ }^{1}$, Niyati Gulati ${ }^{2}$, Sushil Kr. Solanki ${ }^{3}$

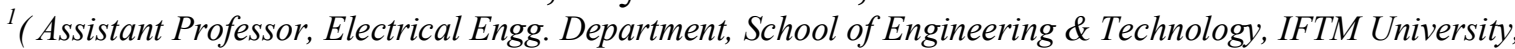 \\ Moradabad, India) \\ 2 (Assistant Professor, Electrical Engg. Department, Moradabad Institute of Technology, Moradabad, India) \\ ${ }_{3}^{3}$ (Assistant Professor (HOD), Electrical \& Electronics Engg. Department, K.N.G.D. MODI ENGG. College, \\ Modinagar)
}

\begin{abstract}
The state estimator processes the information available and produces the best possible estimate of the true state of the system. It is a digital processing scheme which provides a real-time data base for many of the central control and dispatch functions in a power system. The real-time data are processed by state estimator, a computer program which calculates voltage magnitudes and relative phase angles of the system buses. While the state estimator gives result similar to those available from conventional power-flow program, the input data and calculation procedure are quite different. Bus injections along with line flow measurements form the measurement vector in the state estimator in order to evaluate the final states of the power system. Test results without and with measurement noises have been presented. Estimator is also capable of identifying the bad data present in the measurement vector.
\end{abstract}

Keywords: Data Acquisition System, Dynamic State, Estimator and Tracking State.

\section{Introduction}

The growth in size and complexity of electric power systems along with increase in power demand has necessitated the use of modern Energy Management Systems (EMS). In the past few decades, major advances in the hardware and software technologies have transformed the power system control from a simple process control to a system of distributed processing capable of supporting several levels of application functions. The supervisory control and data acquisition/automatic generation control (SCADA/AGC) systems have now given way to the full fledged Energy Management systems. This very large and complex hardware software system is based in utility company's load dispatch or control centres which perform extensive on-line monitoring, assessment, analysis and optimization functions to ensure economical and secure operation of power system as well as to facilitate the periodic tasks carried out by the operating personnel [1]. Data received at energy control centers through telemetry link contains small random noise due to inaccuracy of the meter through which they have been acquired. This could be in Analog/digital conversion circuit and communication link. Moreover, some of the data may be missing due to communication Failure or meter faults or may contain gross error. Since, the success of all the advanced functions being carried out at energy control centers depend on how accurate the system data are being used to run the software. Thus the state estimation of an electric power system is a function which utilizes the statistical criterion to filter out the noise which is usually present in the telemetered measurements acquired through the data acquisition system and which determines the system operating states (complex bus voltages). The output of the state estimator is used as need to various advanced function of the Energy Management. To obtain reliable estimate of the system states, redundant set of measurements are taken and processed [1].

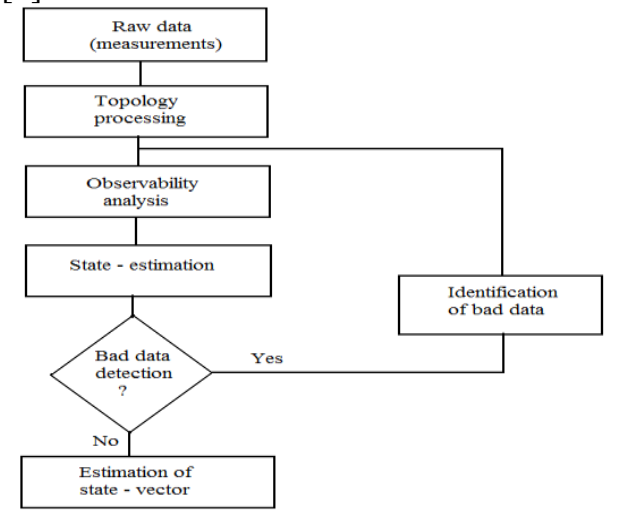

Fig 1.:- Conventional State Estimators 


\section{Type Of State Estimation}

Depending on the time variant or invariant nature of measurements and the static dynamic model of the power system states being utilized, the state estimation can be classified into three categories:
$i$. Static state estimation
ii. $\quad$ Tracking state estimation
iii. Dynamic state estimation

The static state estimation is defined as the data processing algorithm for converting redundant meter readings and other available information in to an estimate of the vector, while measured data are taken to be time invariant and state model of the power system is considered [1-3].

The tracking state estimation algorithms are based on a simple extension of the static state estimation techniques. They utilize the recent available value of the system states to update their estimated values non iteratively during the subsequent sampling period. This class of estimators has been arisen from the natural need of making static state estimators as efficient as possible in regard to the computational speed making them more suitable for real-time implementation [4].

The dynamic state estimator utilizes, in addition to the present states, the previous estimates of the states. The capability of forecasting the state vector one step ahead is an important advantage of the advantage of the dynamic estimators. State prediction gives a longer decision time to the system operator, because economic dispatching, security assessment and other functions can be performed in advance. In dynamic state estimation, dynamic model for the time behaviour of system states is utilized, whereas tracking and static state estimators do not require any dynamic model of the system states. In the present thesis only static state estimation has been studied [5].

\section{Classification Of Error}

Observability analysis determines whether the given numbers of measurements are estimating the state vector. State estimator, computes the voltage magnitude and phase angle at every bus from the available realtime measurements, usually consisting of real and reactive power. Depending on the severity of errors introduced into telemetered measurements, they have been classified in to following three categories [7].

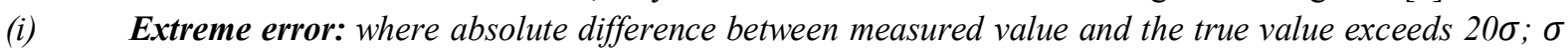
being the standard deviation of measurements.

(ii) Gross error (Bad data): where absolute difference between measured value and the true value is between $5 \sigma$ and $20 \sigma$.

(iii) Normal error: where absolute difference between measured value and true value is less than $5 \sigma$.

The extreme erroneous data are rejected by pre-filtering and measurements. Simple checks on the incoming raw measurements data (e.g. Limit cheeks) can be eliminated certain successively bad data. However, some gross errors will pass through this pre-filtering and enter the estimation process, where there effect on the final solution must be minimized eliminated completely. The gross errors may be due to malfunction of the meter or breakdown of the equipment.

Bad data processor detects the presence of bad data (gross error) in the measurements .after bad data is detected; it identifies which measurements are bad. These are eliminated from the set of measurements to be utilized for the state estimation.

\section{Modelling Of Static State Estimation}

Within energy management systems, state estimation is a key function for building a real-time model. A real-time model is a quasi-static mathematical representation of the current conditions in an interconnected power network. This model is extracted at intervals from snapshots of real-time measurements (both analog and status). The new modelling needs associated with the introduction of new control devices and the changes induced by emerging energy markets are making state estimation and its related functions more important than ever.

\section{Network Real-Time Modelling}

Network real-time models are built from a combination of snapshots of real-time measurements and static network data. Real time measurements consist of analog measurements and statuses of switching devices, whereas static network data correspond to the parameters and basic substation configurations.

The real-time model is a mathematical representation of the current conditions in a power network extracted at intervals from state estimation results. Ideally, state estimation should run at the scanning rate (say, 
at every two seconds). Due to computational limitations, however, most practical estimators run every few minutes or when a major change occurs [8].

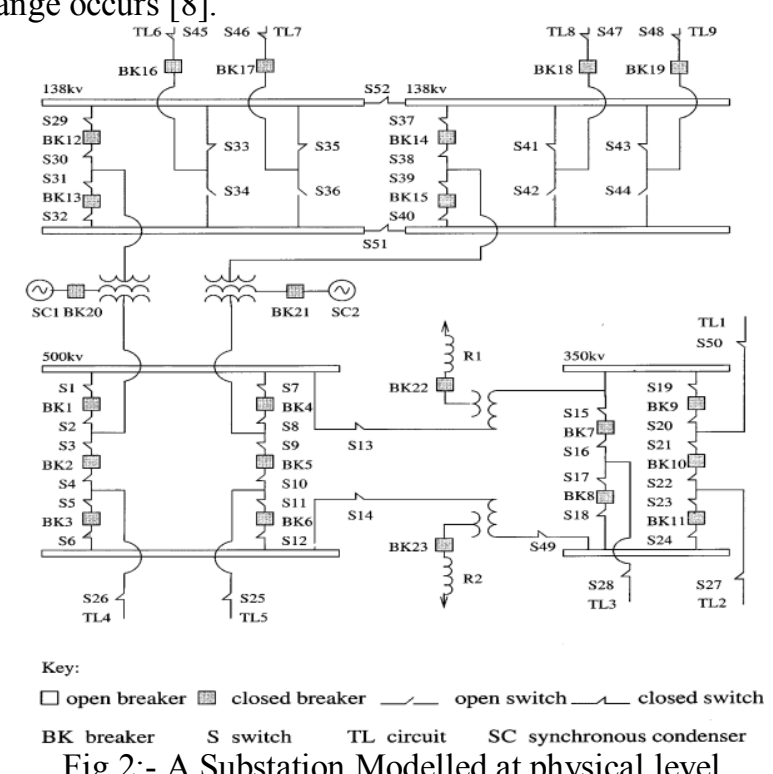

Fig 2:- A Substation Modelled at physical level

\section{Bad Data Processing}

Static-state estimation is related in many ways to conventional load-flow calculations. However, the state estimator is designed to handle the many uncertainties associated with an actual system using meter readings telemetered in real time to a digital computer. Uncertainties arise because of meter and communication errors, incomplete metering, errors in mathematical models, unexpected system changes, etc. The purpose of a static state estimator is to "clean up" the incoming data (actual measurements, system status information, and possibly pseudo measurements) and provide the rest of the control computer or the system operator with a "reliable" set of numbers (the state estimate) which are truly representative of the actual system. Thus there is a great conceptual and practical difference between static-state estimation as a part of a real-time control system and the usual load-flow studies done the office for system planning

\section{Problem Statement}

Static-state estimation is related to conventional load-flow calculations. However, the state estimator is designed to handle the many uncertainties associated with trying to do an on-line load flow for an actual system using meter readings telemetric in real time to a digital computer. Uncertainties arise because of meter and communication errors, incomplete metering, errors in mathematical models, unexpected system changes, etc. These uncertainties make for large differences between the usual load flow studies done in the office for system planning and on-line estimation done as part of a control system. Three possible approaches for obtaining the crucial data base of Fig.2. are:

1) data acquisition with no processing

2) data acquisition with digital computer used for data logging, limit checking, and simple logic comparisons between redundant measurements of essentially the same variable

3) data acquisition with a digital computer used in a systematic way, based on a mathematical model, to clean up the data (by treating small random errors, bad data, modelling errors, and parameter errors), and to compute (estimate) quantities and variables which are not directly measured. The third approach is static state estimation.

\section{Conventional State Estimation}

In conventional state estimation, network real-time modelling is decomposed into: i) the processing of logical data (the statuses of switching devices) and ii) the processing of analog data (e.g., power flow, power injection, and voltage magnitude measurements). During topology processing, the statuses of breakers/switches are processed using a bus section /switching-device network model of the type illustrated in Fig.1. During observability analysis and state estimation, the network topology and parameters are considered as given, and analog data are processed using the bus/branch network model. In the conventional approach, logical data are checked by the topology processor and the analog data are checked by the state estimator [8]. 


\section{Generalized State Estimation}

In generalized state estimation there is no clear-cut distinction between the processing of logical and analog data since network topology processing may include local, substation level, state estimation, whereas when state estimation is performed for the whole network, parts of it can be modelled at the physical level (bus-section/switching-device model). The term generalized is used to emphasize the fact that not only states, but also parts of the network topology, or even parameters, are estimated. The explicit modelling of switches facilitates bad data analysis when topology errors are involved (incorrect status of switching devices). In this case, state estimation is performed on a model in which parts of the network can be represented at the physical level. This allows the inclusion of measurements made on zero impedance branches and switching devices. The conventional states of bus voltages and angles are augmented with new state variables. Observability analysis is extended to voltages at bus sections and flows in switching devices, and if their values can be computed from the available measurements they are considered to be observable.

\section{The Structure And Formation}

The phase angles of the voltages at different substations of the systems cannot be economically measured, but voltage magnitudes are routinely monitored. In order to estimate both voltage angles and magnitudes, we must choose the angle at one of the $\mathrm{N}$ buses of the system as reference for all other angles, which leaves $\mathrm{N}-1$ angles and $\mathrm{N}$ magnitudes to be calculated by Eq. hence the state estimation Jacobian $\mathbf{H}_{\mathbf{x}}$, unlike the square Jacobian $\mathbf{j}$ of convectional Newton-Raphson power flow, always has $(2 \mathrm{~N}-1)$ columns and a larger number $\mathrm{Nm}$ of rows, Each row of $\mathbf{H}_{\mathbf{x}}$ corresponds uniquely to one of the measured quantities indicated in the transmission-line equivalent circuit of fig.3. namely,

$>$ The voltage magnitude $\left|V_{\mathrm{i}}\right|$ at a typical bus ' $\mathrm{i}$ ',

$>$ The active power $\mathrm{P}_{\mathrm{i}}$ injected into the network at bus ' $\mathrm{i}$ ',

$>$ The reactive power $\mathrm{Q}_{\mathrm{i}}$ injected in to the network at bus ' $\mathrm{i}$ ',

$>$ The active power flow $\mathrm{P}_{\mathrm{ij}}$ at bus $\mathrm{i}$ or $\mathrm{P}_{\mathrm{ji}}$ at bus $\mathrm{j}$ in the line connecting buses $\mathrm{i}$ and $\mathrm{j}$,

$>$ The reactive power flow $\mathrm{Q}_{\mathrm{ij}}$ at bus $\mathrm{i}$ or $\mathrm{Q}_{\mathrm{ji}}$ at bus $\mathrm{j}$ in the line connecting buses $\mathrm{i}$ and $\mathrm{j}$. Although linecharging capacitances is shown in Fig.3. line-charging megavars.

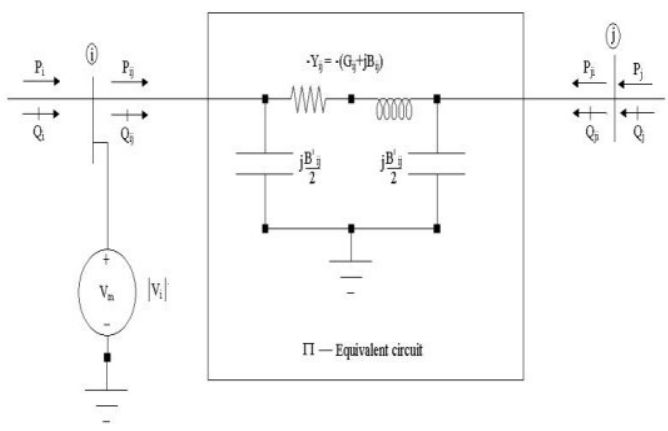

Fig.3: The per-phase representation of transmission line

\section{Least Squares Method}

The electric power transmission system uses wattmeters, varmeters, voltmeters, and current meters to measure real power, reactive power, voltages, and currents, respectively.

These continuous or analog quantities are monitored by current and potential transformers and buses of the power plants and substations of the system. The analog quantities pass through transducers and analogto-digital converters, and the digital outputs are then telemetered to the energy control center over various communication links. The data received at the energy control center is processed by computer to inform the system operators of the present state of the system. The acquired data always contains inaccuracies which are unavoidable since physical measurements (as opposed to numerical calculations) cannot be entirely free of random errors or noise. These errors can be quantified in a statistical sense and the estimated values of the quantities being measured are then either accepted as reasonable or rejected if certain measures of accuracy are exceeded

\section{Weighted Least Squares (Wls) Estimation}

In view of the uncertainties in the measurements in the measurements, the stochastic approach of estimation theory provides a useful tool for determining the system states. In presence of white Gaussian noise the optimal estimate of state variable $\tilde{x}$ is given by that value of $\mathrm{x}$ for which the scalar sum of the weighted 
squares of the measurements errors takes its minimum[1, 2]. Thus the state estimation involves minimizing a function $\mathrm{j}(\mathrm{x})$ defined as,

$$
\mathrm{J}(\mathrm{x})=[\mathrm{z}-\mathrm{h}(\mathrm{x})]^{\mathrm{T}}\left[\mathrm{R}^{-1}\right][\mathrm{z}-\mathrm{h}(\mathrm{x})]
$$

Where, inverse of the noise co-variance matrix $\left[R^{-1}\right]$ is the weighing matrix. Using the WLS method the state vector can be recursively updated as,

$\widehat{\mathrm{x}}^{(\mathrm{q}+1)}=\mathrm{x}^{(\mathrm{q})}+\left[\mathrm{H}^{\mathrm{T}} \mathrm{R}^{-1} \mathrm{H}\right]^{-1}[\mathrm{H}]^{\mathrm{T}}\left[\mathrm{R}^{-1}\right]\left[\mathrm{Z}-\mathrm{h}\left(\mathrm{x}^{(\mathrm{q})}\right)\right]$

Where, $\mathrm{q}$ is the iteration count

$\mathrm{H}$ is the $(\mathrm{m} \times \mathrm{n})$ jacobian matrix defined as,

$\mathbf{H}=\left[\begin{array}{l}\frac{\partial \mathbf{h}_{1}}{\partial \mathbf{x}_{1}} \ldots \ldots \ldots . \frac{\partial \mathbf{h}_{1}}{\partial \mathbf{x}_{\mathbf{n}}} \\ \frac{\partial \mathbf{h}_{\mathbf{m}}}{\partial \mathbf{x}_{1}} \ldots \ldots . . . \frac{\partial \mathbf{h}_{\mathbf{m}}}{\partial \mathbf{x}_{\mathbf{n}}}\end{array}\right]$

And, $\left(H^{T} R^{-1} \mathrm{H}\right)$ is the information matrix.

And above WLS method of static state estimation requires computation of Jacobian matrix $\mathrm{H}$ and inverse of information matrix in each iteration, making the model to be time extensive.

\section{Conclusion}

In this paper, State estimation over view for power system control by load flow program input data and calculation procedure are quite different vector test. Estimator are used for detect the bad data present in the system. And also estimator is capable of identification the bad data present in the measurement vector real-time data base for many of the central control and dispatch function in a power system.

\section{References}

[1] F.C. Schweppe and J.Wildes, "Power System Static State Estimation, Part-I: Exact Model”, IEEE Trans. on Power Apparatus and Systems, Vol. PAS-89, No. 1, pp. 120-125, January 1970.

[2] F.C. Schweppe and D.B.Rom, "Power System Static State Estimation, part-II: Approximation Model", IEEE Trans. on Power Apparatus and Systems, Vol. PAS-89, No. 1, pp. 125-130, January 1970.

[3] F.C. Schweppe, "Power System Static State Estimation, part-III: Implementation", IEEE Trans. On Power Apparatus and Systems, Vol. PAS-89, No. 1, pp. 130-135, January 1970.

[4] R.D.Masicllo and F.C.Schweppe, "A Tracking Static State Estimator", IEEE Trans. on Power Apparatus and Systems, Vol. PAS90, No.3, pp. 1025-1033, May/June 1971.

[5] A.S.Debs and R.E.Larson, "A Dynamic Estimator for Tracking the State of a Power System", IEEE Trans. on Power Apparatus and Systems, Vol. PAS-89, No. 3, pp.1670-1676, Sept. /Oct. 1970.

[6] Felix F.Wu, Wen-Hsiung, "Observability Analysis and Bad Data Processing for State Estimation with Equality Constraints", IEEE Trans. on Power System, Vol. 3, No. 5, pp. 541-548, May 1988

[7] Felix F.Wu, Wen-Hsiung, E.Liu and Shau-Ming lun, "Observability Analysis and Bad Data Processing for State Estimation with Equality constraints", IEEE Trans. on Power Systems, Vol. 3, No.2, pp. 541-548, May 1988.

[8] A.Monticelli, "Electric Power System State Estimation",in the Proceeding of the IEEE, Vol. 88, No. 2, pp. 262-282, February 2000.

[9] Roberto Mq| ${ }^{3}$ nguez Department of Mathematics, University of Castilla-La Mancha Antonio J. Conejo Department of Electrical Engineering University of Castilla-La Mancha. Ali S. Hadi Department of Mathematics American University in Cairo, Non Gaussian State Estimation in Power Systems, International Conference on Mathematical and Statistical Modeling in Honor of Enrique Castillo, June 28-30, 2006.

[10] Vassilis Kekatos, Member, IEEE, and Georgios B. Giannakis, Fellow, IEEE “Distributed Robust Power System State Estimation IEEE transactions on power systems", vol. 28, no. 2, May 2013. 